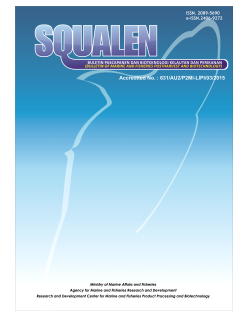

\title{
BIOASSAY-GUIDED ISOLATION OF AN ANTIBACTERIAL COMPOUND FROM THE INDONESIAN SOFT CORAL Sarcophyton trocheliophorum
}

\author{
Masteria Yunovilsa Putra ${ }^{1 *}$, Ahmad Saparhadi ${ }^{1,2}$, Firmansyah Karim ${ }^{1,2}$, \\ Tutik Murniasih', and Respati Tri Swasono ${ }^{2}$
}

${ }^{1}$ Research Center for Oceanography, Indonesian Institute of Science, Jl. Pasir Putih I, Ancol Timur, Jakarta, Indonesia 14430;

2Department of Chemistry, Faculty of Mathematics and Natural Sciences,Universitas Gadjah Mada, Sekip Utara, Yogyakarta, Indonesia

Article history:

Received: 2 May 2016; Revised: 10 July 2016; Accepted: 18 July 2016

\begin{abstract}
With the aim of searching for new antibacterial compound from marine soft corals, the investigation had been conducted on antibacterial activity of an extract from soft corals Sarcophyton trocheliophorum in the $n$-hexane, ethyl acetate, $n$-butanol, and aqueous fractions. The antibacterial activity was tested against two Gram-positive bacteria, viz. Bacillus subtilis (ATCC 6633) and Staphylococcus aureus (ATCC 25923), and two Gram-negative bacteria, viz. Escherichia coli (ATCC 25922) and Vibrio cholerae (ATCC 14035) using the agar disc diffusion assay. Among them, the n-hexane fraction was the most active against three tested bacteria, viz. Bacillus subtilis, Staphylococcus aureus and Vibrio cholerae at the concentration $125 \mu \mathrm{g} / \mathrm{ml}$, with inhibition zone 14.2, 18.2, $13.8 \mathrm{~mm}$, respectively. Isolation and purification of the active component from the n-hexane fraction led to a known cembranoid-type diterpene, sarcophytoxide. The chemical structure of the isolated compound was determined by IR, MS and NMR, as well as compared to data from the literature. Sarcophytoxide showed moderate activity against $B$. subtilis, $S$. aureus and $V$. cholerae, with a minimum inhibitory concentration (MIC) of $125,100,125 \mathrm{mg} / \mathrm{ml}$, respectively.

Keywords: antibacterial, cembranoid, soft coral, Sarcophyton trocheliophorum
\end{abstract}

\section{Introduction}

Soft corals (subclass Octocorallia, order Alcyonacea) are widely distributed along tropic and subtropic oceans. These organisms are known to produce a broad array of chemical compounds, particularly sesquiterpenoid, diterpenoids and steroids. Currently, more than 20 publications have reported on the chemical compounds from Indonesian soft corals such as Lobophytum sp., Cladiella sp., Sarcophyton sp., and Sinularia sp, dominated by the cembrane-type of diterpenes (Putra \& Murniasih, 2016).

Soft corals of the genus Sarcophyton are well recognized as a rich source of secondary metabolites including sterols, sesquiterpenoid, diterpenoids and other related metabolites (Tang, Sun, Zou \& Yin, 2016; Zubair, Al-Footy, Ayyad, Al-Lihaibi \& Alarif, 2015; Yao et al., 2012). Untill now, nearly 16 species of soft corals of the genus Sarcophyton have been chemically investigated from various geographical areas (Chen, Liang, Li, Xiao \& Guo, 2016). Cembranoid-type diterpenes appeared to be characteristic constituents of the genus Sarcophyton and commonly described as chemical defense tools to protect soft corals against natural predators. Cembranoids are considerable interest and merit continuous attention as they exhibit a range of biological activities especially antibacteria, anti-inflammatory and cytotoxic (Ishii, Kamada \& Vairappan, 2016; Lin et al., 2010; Pollastro et al., 2016).

In the frame of our ongoing screening for antibacterial compounds from Indonesian marine soft corals, there was an opportunity to analyze a specimen of the soft coral Sarcophyton trocheliophorum, collected from Selayar Island (South

\footnotetext{
${ }^{*}$ Corresponding author.
}

E-mail: mast001@lipi.go.id 
Sulawesi, Indonesia). The $n$-hexane, ethyl acetate, $n$-butanol, and aqueous fractions Sarcophyton trocheliophorum extract, have been evaluated for antibacterial activity against four pathogen bacteria viz. Bacillus subtilis (ATCC 6633), Staphylococcus aureus (ATCC 25923), Escherichia coli (ATCC 25922) and Vibrio cholerae (ATCC 14035). The $n$-hexane fraction showed the highest antibacterial activity and led to the isolation of a known cembranoid-type diterpene, named sarcophytoxide as antibacterial compounds. The structure of sarcophytoxide was elucidated through extensive spectroscopic analyses including IR, NMR, MS techniques and comparison with literature data. Sarcophytoxide showed inhibitory activity against two Gram-positive bacteria, viz. S. aureus and $B$. subtilis and one Gram-positive bacteria V. cholerae.

\section{Material and Methods}

\subsection{General Experimental Procedures and Chemical Reagents}

${ }^{1} \mathrm{H}(500 \mathrm{MHz})$ and ${ }^{13} \mathrm{C}(125 \mathrm{MHz}) \mathrm{NMR}$ spectra were measured by JEOL spectrometer. Chemical shifts were referenced to the residual solvent signal $\left(\mathrm{CDCl}_{3}: \mathrm{dH} 7.26, \mathrm{dC}\right.$ 77.0). ESIMS spectra were obtained by LC/MS (Waters Alliance 2695). Ethyl acetate, $n$-hexane, $n$-butanol, methanol, and dichloromethane were purchased from Merck (Darmstadt, Germany). All chemicals used were analytical grade. Silica gel 60 (Merck) as the stationary phase for column chromatography and plastic-backed plates coated with Si gel F254 (Merck) were used for TLC. Plates were visualized by spraying with vanillin- $\mathrm{H}_{2} \mathrm{SO}_{4}$ and warming.

\subsubsection{Soft coral samples}

Colonies of soft coral Sarcophyton trocheliophorum were collected by scuba diving in Selayar Island, South Sulawesi, Indonesia at a depth $10 \mathrm{~m}$ in June 2015. A voucher specimen was deposited in the Research Center for Oceanography-LIPI, under the registration number SLYR SC-2.

\subsubsection{Extraction and Isolation}

The colonies of Sarcophyton trocheliophorum (225 g) were repeatedly extracted with $\mathrm{MeOH}: \mathrm{CH}_{2} \mathrm{Cl}_{2}(1: 1)$ at room temperature to get the crude extract $(5.2 \mathrm{~g})$. The crude extract was partitioned based on the polarity of the solvent using $n$-hexane (non-polar), ethyl acetate (semi-polar), n-butanol (polar) and water. Each of fractions was subjected to preliminary phytochemical screening and antibacterial activity. The most active fraction was subjected to further purification using $\mathrm{SiO}_{2}$ gel column chromatography eluted with a gradient system of increasing polarity from $n$-hexane to EtOAc to $\mathrm{MeOH}$. Each of fractions $\mathrm{SiO}_{2}$ gel column chromatography was evaluated for their antibacterial activity. Once fraction ST 2 showed potential antibacterial activity, then it will be further purified by $\mathrm{SiO}_{2}$ column chromatography, eluted with $n$-Hexane: EtOAc (9:1) to afford sarcophytoxide (1.2 g).

\subsection{Chemical Screening}

All fractions were subjected to preliminary phytochemical screening test for the presence of secondary metabolites utilizing the standard conventional protocol described by Senguttuvan et al., 2014.

\subsection{Antibacterial Assay}

\subsubsection{Agar diffusion test}

Antibacterial activity of all fractions from crude extract of Sarcophyton trocheliophorum was conducted using agar disk diffusion method. Briefly, $125 \mu \mathrm{g} / \mathrm{ml}$ the sample was diluted in $\mathrm{MeOH}$ then about $20 \mu \mathrm{l}$ was dropped into $6 \mathrm{~mm}$ diameter filter paper disc. The paper disc was then placed on a Mueller Hinton Agar (Himedia) in a petri dish that had been inoculated with test bacteria. Test bacteria used in this research, were two Gram-negative bacteria (Escherichia coli ATCC 25922, Vibrio cholerae ATCC 14035), two Gram-positive bacteria (Staphylococcus aureus ATCC 25923, Bacillus subtilis ATCC 6633). Inhibition of bacterial growth activity appeared as a clear zone around the paper disc. The inhibition zone was observed after incubation at $37^{\circ} \mathrm{C}$ for $20-24 \mathrm{~h} .10$ $\mu \mathrm{g} / \mathrm{ml}$ ampicillin and methanol were used as a positive and solvent control, respectively. All the assays were performed in triplicate.

\subsubsection{Microdilution method}

The microdilution method was used to evaluate the minimum inhibitory concentration (MIC) of the bioactive compound which showed good activity (growth inhibition zone more than $9 \mathrm{~mm}$ ) in the disc diffusion assay. Tests were performed in 96-well round bottom sterile culture plates using the Infinite ${ }^{\circledR} 200$ PRO microplate reader (Tecan Austria $\mathrm{GmbH}$ ). The assay plates were filled with Mueller-Hinton broth medium (MHB) in serial dilutions ranging from 6.25 , $12.5,25,50,100,200,250,500$ and $1000 \mathrm{mg} / \mathrm{ml}$ of isolated compound, ampicillin or methanol and the test microorganism $\left(10^{7} \mathrm{CFU} / \mathrm{ml}\right)$. The turbidity in each 
Table 1. Chemical analysis of all fractions from the crude extract of Sarcophyton trocheliophorum

\begin{tabular}{llcccc}
\hline \multirow{2}{*}{ No } & Chemical constituents & \multicolumn{5}{c}{ Fractions } \\
\cline { 2 - 6 } & & n-Hexane & $n$-Butanol & Ethyl acetate & Aqueous \\
\hline 1 & Alkaloids & + & - & + & + \\
2 & Steroids & + & + & + & - \\
3 & Flavanoids & - & - & - & - \\
4 & Saponins & - & - & + & + \\
5 & Terpenoids & + & + & + & - \\
6 & Phenols & + & + & + & - \\
7 & Tannins & - & - & - & - \\
\hline
\end{tabular}

well was measured at $600 \mathrm{~nm}$, after $24 \mathrm{~h}$ incubation periods at $37^{\circ} \mathrm{C}$.

\section{Results and Discussion}

Chemical constituents from the crude extract of Sarcophyton trocheliophorum were showed in Table 1. In the present study, the chemical analysis of all fractions indicated the presence of alkaloids, steroids, triterpenoids, saponins, terpenoids, and phenols. The $n$-hexane, ethyl acetate, and $n$-butanol fractions from Sarcophyton trocheliophorum crude extract showed positive results for terpenoids and steroids. The presence of secondary metabolites in the fractions can provide a preliminary explanation of their antibacterial activities. Numerous articles reported the isolation of terpenoid compounds from the soft coral genus Sarcophyton, which displayed good biological activities such as anti-inflammatory, antibacterial and cytotoxic activity. Most of the isolated terpenoids were cembranoid-type diterpenes, which were found in high concentrations (up to $5 \%$ dry weight) in soft corals (Gross et al., 2003; Coll, 1992).

All fractions from Sarcophyton trocheliophorum crude extract showed antibacterial activity against Gram-positive (B.subtilis dan S. aureus) and Gramnegative (E. coli dan V. chloreae) bacteria (Figure. 1). The $n$-hexane fraction showed strong inhibition activity against $B$. subtilis, $S$. aureus, and $V$. chloreae, with inhibition zones of $14.2,18.2$, and $13.8 \mathrm{~mm}$, respectively, while other fractions showed moderate activity against all tested bacteria. Differences were observed in the antibacterial activities of the fractions.
These could be due to the differences in their chemical structures as well as the mechanism of activity of their bioactive constituents.

Furthermore, $n$-Hexane fraction, which shown potential antibacterial activity, has been purified by $\mathrm{SiO}_{2}$ gel column chromatography using a gradient system of increasing polarity from $n$-hexane to EtOAc to $\mathrm{MeOH}$. All fractions were analyzed by TLC, and those with similar profiles were combined to give fractions ST-1 to ST-21. Each of these fractions was evaluated for their antibacterial activity. The results showed that fraction ST-2 has a potential inhibitory activity against $S$. aureus, $B$. subtilis, and $V$. cholerae. This fraction was subsequently subjected to $\mathrm{SiO}_{2}$ column chromatography, eluted with $n$-Hexane EtOAc (9:1), to afford a known cembranoid-type diterpene, sarcophytoxide (Figure 2). As a pure compound, this compound yielded $1.2 \mathrm{~g}$ as yellow crystalline needles.

The molecular formula of the compound was determined to be $\mathrm{C}_{20} \mathrm{H}_{30} \mathrm{O}_{2}$ from the El-MS (positive ions), with the molecular ion peak at $\mathrm{m} / \mathrm{z} 325[\mathrm{M}+\mathrm{Na}]^{+}$ for $\mathrm{C}_{20} \mathrm{H}_{30} \mathrm{O}_{2} \mathrm{Na}$. The IR spectrum showed absorptions at $2930,2851,1664,1446,1383,1035,940$ and 866 $\mathrm{cm}^{-1}$. ${ }^{1} \mathrm{H}\left(500 \mathrm{MHz}, \mathrm{CDCl}_{3}, \mathrm{~J}\right.$ in $\left.\mathrm{Hz}\right): 5.54(\mathrm{~m}, 1 \mathrm{H}, \mathrm{H}-$ 2), $5.22(\mathrm{~d}, 1 \mathrm{H}, J=10.2 \mathrm{~Hz}, \mathrm{H}-3), 5.09$ (dd, $1 \mathrm{H}, J=$ 10.8, 5.1 Hz, H-11). 4.49 (s, 1H, H-16), 2.71 (t, 1H, J $=4.1, \mathrm{H}-7), 2.6(1 \mathrm{H}$, overlapped, $\mathrm{H}-14 \mathrm{~b}), 2.3(1 \mathrm{H}$, overlapped $\mathrm{H}-5)$, $2.2(1 \mathrm{H}$, overlapped, $\mathrm{H}-10 \mathrm{a}), 2.0(1 \mathrm{H}$, overlapped, $\mathrm{H}-9 \mathrm{a}), 1.9(1 \mathrm{H}$, overlapped, $\mathrm{H}-13), 1.9(1 \mathrm{H}$, overlapped, $\mathrm{H}-10 \mathrm{~b}), 1.9$ (1H, overlapped, $\mathrm{H}-6 \mathrm{a}), 1.81$ (s, 3H, H-18), $1.64(\mathrm{~s}, 3 \mathrm{H}, \mathrm{H}-17), 1.6(1 \mathrm{H}$, overlapped, $\mathrm{H}-14 \mathrm{~b}), 1.58(\mathrm{~s}, 3 \mathrm{H}, \mathrm{H}-20), 1.3(1 \mathrm{H}$, overlapped, $\mathrm{H}-$ 6b), 1.26 (s, 3H, H-19) $1.0(\mathrm{dt}, 1 \mathrm{H}, J=13.0,2.9, \mathrm{H}-$ 


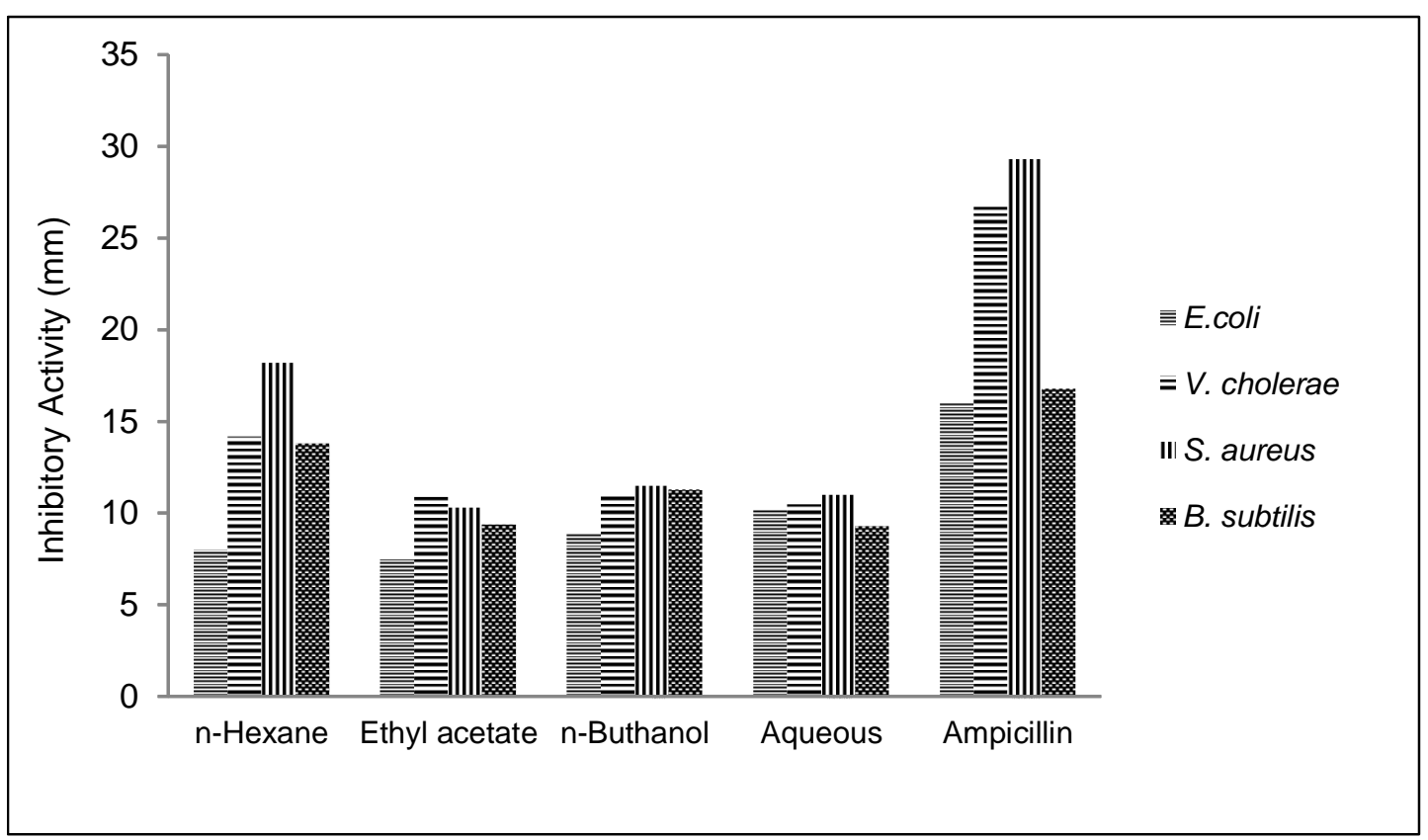

Figure 1. Antibacterial assay of all fractions from the $\mathrm{MeOH}-\mathrm{CH}_{2} \mathrm{Cl}_{2}$ extract of Sarcophyton trocheliophorum using an agar disc diffusion method.

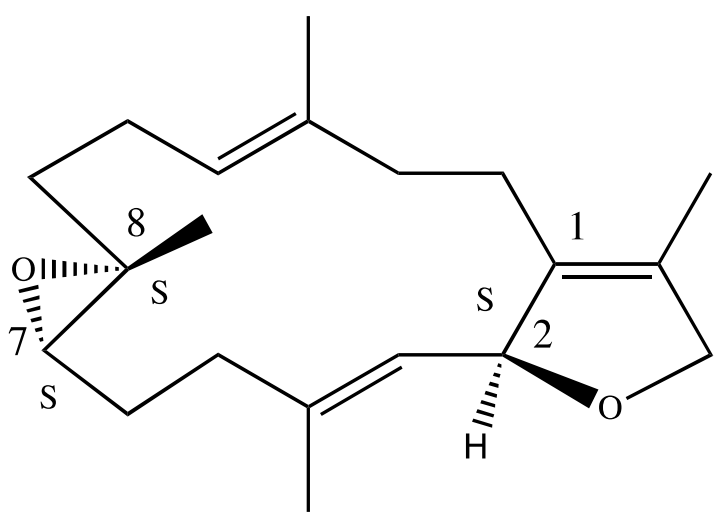

Figure 2. Sarcophytoxide.

9) $;{ }^{13} \mathrm{C}\left(125 \mathrm{MHz}, \mathrm{CDCl}_{3}\right): 139.2$ (C-4), 136.7 (C-12), 133.2 (C-1), 127.8 (C-15), 126.3 (C-3), 126.3 (C-11), 83.6 (C-2), 78.3 (C-16), 61.9 (C-7), 59.8 (C-8), 39.7 (C-9), 37.6 (C-5), 36.7 (C-13), 26.0 (C-14), 25.4 (C-6), 23.5 (C-10), 17.0 (C-19), 15.6 (C-18), 15.2 (C-20), 10.1 (C-17). Based on the physicochemical analyses, including IR, 1D NMR, MS, and their comparisons with data from the literature, the isolated compound was determined to be a known cembranoid typediterpene, $(2 S, 7 S, 8 S)$-sarcophytoxide (Bowden, Coll, Heaton, Konig \& Scheuer, 1987; Nii et al., 2006; Pollastro et al., 2016).

A $(2 S, 7 S, 8 S)$-sarcophytoxide was screened for antibacterial activity using agar disk diffusion. The results of the antibacterial activity of sarcophytoxide shown in Table 3 displayed potential inhibitory activity against $S$. aureus, $B$. subtilis, and $V$. cholerae. Further characterization of the antibacterial properties of sarcophytoxide by determining the MICs using the broth microdilution method was done (Table 3 ). In this work, sarcophytoxide showed moderate activity against two Gram-positive bacteria ( $S$. aureus and $B$. subtilis) and actively against the Gram-negative bacteria $V$. cholera. It is known that Gram-negative bacteria are less sensitive to marine invertebrate extracts of subtropical or tropical species, while the Gram-positive bacteria were particularly sensitive (Fleury, Coll \& Sammarco, 2006). 
Table 3. Agar plate diffusion assay (zone inhibition in $\mathrm{mm}$ ) and minimum inhibitory concentration of sarcophytoxide and ampicillin (MIC in $\mathrm{mg} / \mathrm{ml}$ )

\begin{tabular}{lcccc}
\hline \multirow{2}{*}{ Microorganisms } & \multicolumn{2}{c}{ Sarcophytoxide } & \multicolumn{2}{c}{ Ampicillin } \\
\cline { 2 - 5 } & $\begin{array}{l}\text { Zone of inhibition } \\
\text { in } \mathbf{~ m m}\end{array}$ & MIC in $\mathbf{~ m g / m l}$ & $\begin{array}{c}\text { Zone of inhibition } \\
\text { in } \mathbf{~ m m}\end{array}$ & MIC in $\mathbf{~ m ~} \mathbf{m l}$ \\
\hline S. aureus & 20.83 & 100 & 25.6 & 25 \\
B. subtilus & 19.58 & 125 & 26.1 & 25 \\
E. coli & 6.95 & - & 24 & - \\
V. cholerae & 19.68 & 125 & 25.3 & 25 \\
\hline
\end{tabular}

\section{Conclusion}

Results of the present investigation showed that the $n$-hexane fraction from Sarcophyton trocheliophorum extract had potential antibacterial activity. Through bioassay-guided fractionation, a cembranoid-type diterpene, named $(2 \mathrm{~S}, 7 \mathrm{~S}, 8 \mathrm{~S})$ sarcophytoxide was isolated from this soft coral. In addition, its chemical structure was identified on the basis of its spectroscopic data and their comparisons with data from the literature. Sarcophytoxide showed moderate activity against two $S$. aureus $B$. subtilis and $V$. cholera. Furthermore, it is known that soft corals of the genus Sarcophyton has secondary metabolites such as sarcophytoxide causing allelopathic effects.

\section{Acknowledgment}

This work was supported by a research grant from the Coral Reef Rehabilitation and Management Program-Coral Triangle Initiative (COREMAP CTI-LIPI 2016 No. 10876401/ ADB LOAN No. 3094 - INO), Indonesian Institute of Sciences awarded to Masteria Yunovilsa Putra and Tutik Murniasih.

\section{References}

Bowden, B. F., Coll, J. C., Heaton, A., Konig, G., \& Scheuer, P.J.J. (1987). The structures of four isomeric dihydrofurancontaining cembranoid diterpenes from several species of soft coral. Nat. Prod. 650" 659.

Chen, W. T., Liang, L. F., Li, X. W., Xiao, W., \& Guo, Y. W. (2016). Further New Highly Oxidative Cembranoids from the Hainan Soft Coral Sarcophyton trocheliophorum. Nat. Prod. Bioprospect., 97-102.
Coll, J. C. (1992). The chemistry and chemical ecology of octocorals (Coelenterata, Anthozoa, Octocorallia). Chem. Rev. 92: 613-631.

Fleury, B. G., Coll, J. C., \& Sammarco, P. W. (2006). Complementary (secondary) metabolites in a soft coral: sex-specific variability, inter-clonal variability, and competition. Mar. Ecol.27, 204-218.

Gross, H., Kehraus, S., Nett, M., König, G. M., Beil, W., \& Wright, A. D. (2003). New cytotoxic cembrane based diterpenes from the soft corals Sarcophyton cherbonnieri and Nephthea sp. Org. Biomol. Chem., 1,944-949.

Ishii, T., Kamada, T., \& Vairappan, C. S. (2016). Three new cembranoids from the Bornean soft coral Nephthea sp. J. Asian. Nat. Prod. Res., 18(5), 415422.

Lin, W., Su, J., Lu, Y., Wen, Z., Dai, C., Kuo, Y., \& Sheu, J. (2010). Cytotoxic and anti-inflammatory cembranoids from the Dongsha Atoll soft coral Sarcophyton crassocaule. Bioorg. Med. Chem., 18,1936-1941.

Nii, K., Tagami, K., Matsuoka, K., Munakata, T., Ooi, T., \& Kusumi, T. (2006). Unusual transannular cyclization products of sarcophytoxide, a 14-membered marine cembranoid: Anomalous stereochemistry of epoxideketone rearrangement. Org. Lett., 8(14):, 2957-2960.

Pollastro, F., Golin, S., Chianese, G., Putra, M. Y., Schiano Moriello, A., De Petrocellis \& Appendino, G. (2016). Neuroactive and nti-inflammatory frankincense cembranes: A structure-activity study. J. Nat Prod., 79(7):1762-1768.

Putra, M.Y., \& Murniasih, T. (2016). Distribution and diversity of marine natural products from Indonesian marine organisms. J. Coast. Life. Med.,4(2), 104-107

Sengunttuvan, J., Paulsamy, S., \& Karthika, K. (2014). Phytochemical analysis and evaluation of leaf and oot parts of the medicinal herb, Hypochaeris radicata L. for in vitro antioxidant activities. Asian. Pac. J. Trop. Biomed:; 2014 4(1), 359-367. 
Tang, G. H., Sun, Z. H., Zou, Y. H., \& Yin, S. (2016). New cembrane-type diterpenoids from the South China Sea soft coral Sarcophyton ehrenbrgi. Molecules, 21(5),-587/1-587/7.

Yao, LG, Zhang, HY, Liang, LF, Guo, XJ, Mao, SC, \& Guo, YW. (2012). Yalongenes $A$ and $B$, two new cembranoids with cytoprotective effects from the hainan soft coral Sarcophyton trocheliophorum M arenzeller. Helv. Chim. Acta 95: 235-239.

Zubair, M. S, Al-Footy, K. O., Ayyad, S. E., Al-Lihaibi, S.S., \& Alarif, W.M. (2016). A review of steroids from Sarcophyton species. Nat. Prod. Res., 30(8),: 869-879. 